\title{
An equivalent lumped circuit model for on-chip helical transformers
}

\author{
Wanghui Zou ${ }^{1 \text { a) }}$, Jin $\mathrm{Hu}^{2}$, Diping Chen ${ }^{2}$, and Yun Zeng ${ }^{2}$ \\ ${ }^{1}$ School of Physics and Electronics, Changsha University of Science \\ and Technology, Changsha, Hunan 410114, China \\ ${ }^{2}$ School of Physics and Microelectronics, Hunan University, \\ Changsha, Hunan 410082, China
}

a)zouwh@csust.edu.cn

\begin{abstract}
This paper presents a compact equivalent lumped circuit model for on-chip helical transformers. From fundamental model, a simple coupled $R L$ loop is added to account for the skin and proximity effect, and a series $R L C$ branch is added to account for the parasitic coupling effects between inductors. Several helical transformers with various design configurations were fabricated using a standard $0.18 \mu \mathrm{m} 1 \mathrm{P} 6 \mathrm{M}$ CMOS process. It's demonstrated that the proposed model shows good accuracy over two times selfresonance frequency, which is essential for the design of high-speed and radio-frequency circuit.
\end{abstract}

Keywords: on-chip helical transformer, equivalent lumped circuit model, standard CMOS process

Classification: Electron devices, circuits and modules

\section{References}

[1] N. Shiramizu, et al.: "Scalable transformer model based on ladder topological equivalent circuit for Si RFICs," SiRF (2010) 12 (DOI: 10.1109/SMIC.2010. 5422954).

[2] C. Wang, et al.: "A physics-based equivalent-circuit model for on-chip symmetric transformers with accurate substrate modeling," IEEE Trans. Microw. Theory Techn. 57 (2009) 980 (DOI: 10.1109/TMTT.2009.2014479).

[3] Z. Gao, et al.: "A broadband and equivalent-circuit model for millimeter-wave on-chip m:n six-port transformers and baluns," IEEE Trans. Microw. Theory Techn. 63 (2015) 3109 (DOI: 10.1109/TMTT.2015.2466549).

[4] W. Z. Chen, et al.: "Three-dimensional fully symmetric inductors, transformer, and balun in CMOS technology," IEEE Trans. Circuits Syst. I, Reg. Papers 54 (2007) 1413 (DOI: 10.1109/TCSI.2007.899628).

[5] A. Zolfaghari, et al:: "Stacked inductors and transformers in CMOS technology," IEEE J. Solid-State Circuits 36 (2001) 620 (DOI: 10.1109/4. 913740).

[6] O. El-Gharniti, et al.: "Modelling and characterization of on-chip transformers for silicon RFIC,” IEEE Trans. Microw. Theory Techn. 55 (2007) 607 (DOI: 10.1109/TMTT.2007.893647).

[7] W. Zou, et al:: "An improved analytical series resistance model for on-chip stacked inductors,” ASICON (2013) 1 (DOI: 10.1109/ASICON.2013.6811923). 
[8] Y. Tang, et al: "Broadband compact model for on-chip mm-wave transformers and baluns with emphasis on capacitive coupling effects," IEEE CICC (2011) 1 (DOI: 10.1109/CICC.2011.6055342).

[9] T. E. Kolding: "On-wafer calibration techniques for giga-hertz CMOS measurements," ICMTS (1999) 105 (DOI: 10.1109/ICMTS.1999.766225).

[10] H. Greenhouse: "Design of planar rectangular microelectronic inductors," IEEE Trans. Parts Hybrids Packag. 10 (1974) 101 (DOI: 10.1109/TPHP.1974. 1134841).

\section{Introduction}

On-chip transformers, or coupled inductors, are often used for the design of highspeed and radio-frequency circuit blocks, such as voltage-controlled oscillator, high-bandwidth amplifier, impedance matching, and so forth. Usually, the planar inductors are employed due to their relatively higher quality factor. However, the multilayer inductors, or stacked inductors, show higher inductance density, which means they occupy less area for the same inductance. As the integrated circuits technologies advance, more metal layers are available for the design of multilayer inductors, and make it possible that the devices can be more compact. As one of the most important work, the modeling of planner transformers drew much research effort $[1,2]$. However, because of their complexity and diversity, the modeling of multilayer transformers is not that easy. In [3], a lumped model was proposed for stacked transformer, which may not applicable to the other multilayer transformers. In [4], a distributed model was proposed for symmetric multilayer transformers. Though physical and universal to some extent, it's not convenient for practical design due to too many parameters needed.

Helical inductor, as the simplest multilayer inductor, has only one turn on each layer, and then the number of turns equals to the number of layer used. A helical transformer consists of two coupled helical inductors. Fig. 1a shows the 3D-view of helical transformer. For multilayer inductors, because the space between metal layers is small, and the turn width is always large, the parasitic capacitance seems significant. However, according to the analysis in [5], it can be verified that the equivalent capacitance across the two terminals of helical inductor is actually small, and if more layers are stacked, the final equivalent capacitance gets even smaller. This is why the helical inductor possesses high self-resonance frequency $f_{\mathrm{SR}}$ along with high inductance density. The helical transformer will also have this benefit. In this paper, we discuss the modeling of helical transformer. A compact equivalent lumped circuit model for helical transformer is given, which shows good agreement with the measurement results.

\section{Device structure and circuit model}

We designed the helical transformers in a standard 0.18 um CMOS process, which provides 6 metal layers. As shown in Fig. 1a, the inner and outer inductors are placed in concentric style. The inner inductor uses six metal layers, from M1 to M6, while the outer inductor uses four metal layers, from M2 to M5, to facilitate the routing of lead wires for the inner inductor. 


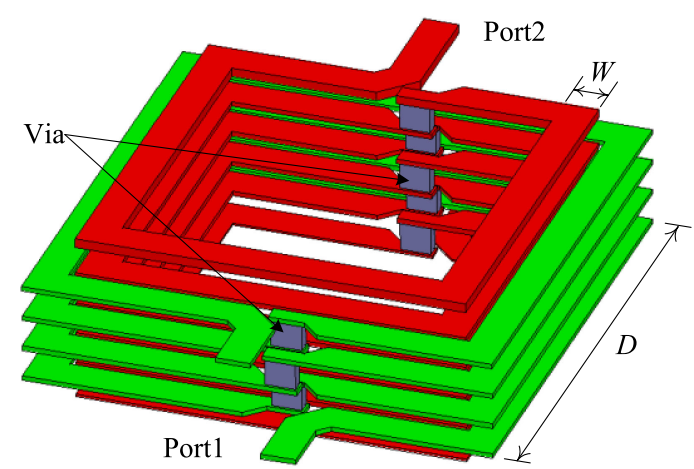

(a)

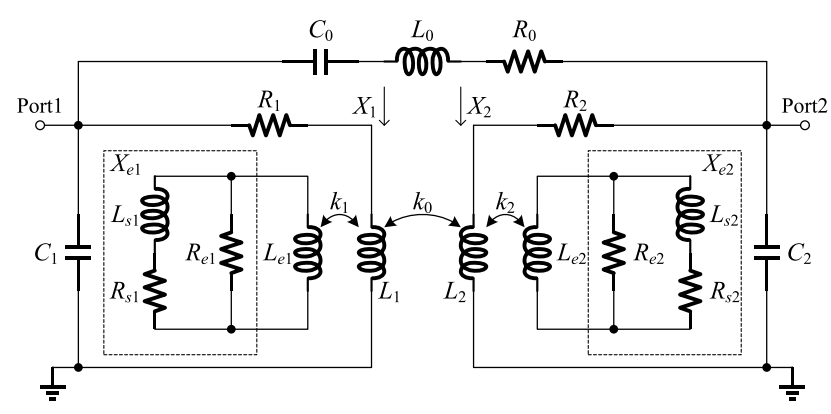

(b)

Fig. 1. (a) 3-D view ( $D$ : outer diameter, $W$ : turn width) and (b) proposed equivalent lumped circuit model of helical transformer. The lower terminals of both two inductors are grounded.

Fig. 1b shows the proposed equivalent lumped circuit model for helical transformer. $L_{1}, R_{1}, C_{1}, L_{2}, R_{2}, C_{2}$ and $k_{0}$ constitute the fundamental model of the transformer [6]. $L_{1}, R_{1}$ can be viewed as dc inductance and dc resistance of the outer inductor, respectively. $C_{1}$ represents the equivalent parasitic capacitance across the two terminals of the inductor, as defined in [5]. $L_{2}, R_{2}$ and $C_{2}$ represent the corresponding parameters of the inner inductor. $k_{0}$ is the coupling coefficient between the outer and the inner inductors. In reality, the inductance and the resistance are frequency dependent due to the skin and the proximity effects. Therefore, a circuit model with the capability to mimic the frequency behavior of the actual inductor is required. In previous papers, a $R L$ ladder structure is proved to be an effective way to capture the frequency behavior of inductor, and also transformer [2, 3]. However, as for a $R L$ ladder, there is no explicit component that can be viewed as dc inductance, and each inductance component is larger than $\mathrm{dc}$ inductance. Thus, when considering the coupling coefficient $k_{0}$, one will very likely obtain a deviated value, which is usually misleading. To make it more physical and meaningful, we employ a coupled $R L$ loop structure as shown in the figure. The final extraction results will reveal that the extracted $k_{0}$ approach the theoretical values.

According to [7], the eddy current in metal path contributes an important part of the aforementioned frequency dependence. As shown in [7], the electro-magnetic field $B$ will penetrate through the metal path and excite eddy current loop, which is always crowed along the path edges. Hence, a $R L$ loop, including the loop resistance and inductance $R_{e 1}$ and $L_{e 1}$, models this loop, and $k_{1}$ models the 
interaction between the main inductance and the loop. Actually, an additional branch, including $R_{s 1}$ and $L_{s 1}$, is also necessary to capture the frequency dependence of the loop resistance. $L_{e 2}, R_{e 2}, L_{s 2}$ and $R_{s 2}$ represent the corresponding parameters of the inner inductor. A more complicated high-order $R L$ loop was discussed in [2]. However, it's found that the $R L$ loop here is accurate enough for the modeling of helical transformer.

Another important part is the parasitic coupling between the inner and the outer inductors. In most previous circuit models, only the parasitic capacitance had been taken into consideration $[2,3]$. However, due to the distributed nature of the coupling effect, the capacitor-only scheme cannot ensure model accuracy at higher frequencies. In [8], an improved scheme was proposed to account for this distributive parasitic coupling effect for planer transformer. A series $R L C$ branch instead of a single capacitor was used. It's believed that this improved scheme is also applicable to helical transformer. Thus, a series $R L C$ branch, including $C_{0}, L_{0}$ and $R_{0}$, is employed as shown in the proposed model.

For a helical inductor, the quality factor of the lower terminal is usually very small due to large parasitic terminal-to-substrate capacitance. Hence, the lower terminals of the inner and the outer inductors are most likely connected to ground in practical circuit design. As a result, the lowest metal actually works like a shielding for the upper metal layers from the substrate, and the substrate network is unnecessary and not considered in the proposed model.

\section{Measurement and model extraction}

Three square helical transformers were fabricated. Fig. 2 shows the chip photo of each device and corresponding design parameters, the outer diameter $D$ and turn width $W$. The spacing between the inner and the outer inductors is fixed at $2 \mu \mathrm{m}$. Because the lower terminal of each port is grounded, the helical transformer is actually a two-port device. Hence, the two-port scattering parameters ( $S$-parameter) are measured on the probe station. After measurement and de-embedding [9], a fitting process was applied to extract model parameters. Generally, it is important to make an appropriate initial guess for each model parameter in order to reach a reasonable and accurate fitting result.

As stated above, $L_{1}$ and $L_{2}$ are viewed as the dc inductance, so the classical Greenhouse's method is effective to make an initial guess [10]. And actually, the mutual inductance, hence the coupling coefficient $k_{0}$, can also be estimated by the Greenhouse's method. $R_{1}$ and $R_{2}$ are viewed as dc resistance and easy to be obtained with the square resistances of the metal. For $C_{1}$ and $C_{2}$, the analytical method in [5] is proved to be accurate for stacked inductors, including helical inductors. For the coupled $R L$ loop, according to the circuit model in Fig. 1b, the impedance $X_{1}\left(X_{2}\right)$ from the upper terminal of $L_{1}\left(L_{2}\right)$ without considering the effect of $k_{0}$ can be expressed as

$$
X_{1,2}=j \omega L_{1,2}+\frac{\omega^{2} k_{1,2}^{2} L_{1,2}}{X_{e 1, e 2} / L_{e 1, e 2}+j \omega}
$$

where $X_{e 1}\left(X_{e 2}\right)$ is the impedance of the dashed box and $\omega$ is the angular frequency. From the equation, it can be found that $X_{e 1}\left(X_{e 2}\right)$ and $L_{e 1}\left(L_{e 2}\right)$ are correlated. That 
is to say, only one parameter is independent and needs to be determined. Hence, we can fix $L_{\mathrm{e} 1}$ and $L_{\mathrm{e} 2}$, and let $X_{e 1}$ and $X_{e 2}$ go through the fitting process. In this paper, we choose $1 \mathrm{nH}$ for $L_{e 1}$ and $L_{e 2}$. For $C_{0}, L_{0}$ and $R_{0}$, though the equations in [8] are proposed for planer transformer, they are still feasible qualitative estimations for helical transformer.

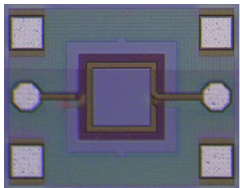

T1: $D=200, W=15$

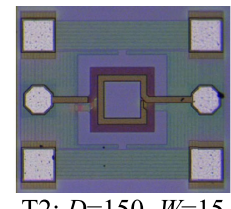

$\mathrm{T} 2: D=150, W=15$

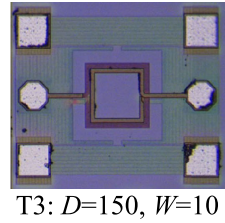

T3: $D=150, W=10$

Fig. 2. Chip photos of the devices with various design parameters (unit: $\mu \mathrm{m}$ ). The spacing between the inner and the outer inductors is $2 \mu \mathrm{m}$.

Table I. Extracted model parameters of fabricated transformers

\begin{tabular}{c|c|c|c}
\hline & $\mathrm{T} 1$ & $\mathrm{~T} 2$ & $\mathrm{~T} 3$ \\
\hline$R_{1}$ & $12.37 \Omega$ & $9.216 \Omega$ & $14.42 \Omega$ \\
\hline$L_{1}$ & $6.661 \mathrm{nH}$ & $4.400 \mathrm{nH}$ & $5.167 \mathrm{nH}$ \\
\hline$C_{1}$ & $136.6 \mathrm{fF}$ & $101.3 \mathrm{fF}$ & $76.56 \mathrm{fF}$ \\
\hline$L_{e 1}$ & $1 \mathrm{nH}$ & $1 \mathrm{nH}$ & $1 \mathrm{nH}$ \\
\hline$R_{e 1}$ & $21.87 \Omega$ & $24.84 \Omega$ & $28.94 \Omega$ \\
\hline$R_{s 1}$ & $35.51 \Omega$ & $34.68 \Omega$ & 50.17 \\
\hline$L_{s 1}$ & $2.998 \mathrm{nH}$ & $2.590 \mathrm{nH}$ & $4.186 \mathrm{nH}$ \\
\hline$k_{1}$ & 0.460 & 0.500 & 0.422 \\
\hline$R_{2}$ & $14.93 \Omega$ & $10.08 \Omega$ & $17.27 \Omega$ \\
\hline$L_{2}$ & $10.97 \mathrm{nH}$ & $6.267 \mathrm{nH}$ & $8.484 \mathrm{nH}$ \\
\hline$C_{2}$ & $86.90 \mathrm{fF}$ & $59.47 \mathrm{fF}$ & $50.74 \mathrm{fF}$ \\
\hline$L_{e 2}$ & $1 \mathrm{nH}$ & $1 \mathrm{nH}$ & $1 \mathrm{nH}$ \\
\hline$R_{e 2}$ & $66.48 \Omega$ & $147.8 \Omega$ & $99.29 \Omega$ \\
\hline$R_{s 2}$ & $61.56 \Omega$ & $57.14 \Omega$ & $105.2 \Omega$ \\
\hline$L_{s 2}$ & $2.696 \mathrm{nH}$ & $2.375 \mathrm{nH}$ & $4.021 \mathrm{nH}$ \\
\hline$k_{2}$ & 0.433 & 0.497 & 0.421 \\
\hline$C_{0}$ & $50.37 \mathrm{fF}$ & $40.32 \mathrm{fF}$ & $35.69 \mathrm{fF}$ \\
\hline$L_{0}$ & $1.393 \mathrm{nH}$ & $0.6396 \mathrm{nH}$ & $1.002 \mathrm{nH}$ \\
\hline$R_{0}$ & $17.85 \Omega$ & $12.52 \Omega$ & $21.93 \Omega$ \\
\hline$k_{0}$ & 0.566 & 0.517 & 0.586 \\
\hline & & &
\end{tabular}

With proper initial guess, the final fitting process converges fast. Table I lists the extracted parameters of all three devices. It's worth noting that the initial calculated $k_{0}$ for the three devices are $0.583,0.522$ and 0.602 , respectively, which are close to the final extracted values. Fig. 3 shows the measured data and the final fitted curves of all three devices. The port inductance and quality factor are calculated as:

$$
\begin{aligned}
L_{\mathrm{p} 1}=\operatorname{imag}(Z 11) / \omega, & L_{\mathrm{p} 2}=\operatorname{imag}(Z 22) / \omega \\
Q_{\mathrm{p} 1}=\operatorname{imag}(Z 11) / \operatorname{real}(Z 11), & Q_{\mathrm{p} 2}=\operatorname{imag}(Z 22) / \operatorname{real}(Z 22)
\end{aligned}
$$

where the subscript of "p1" and "p2" indicate Port1 and Port2, respectively, and $Z 11$ and $Z 22$ are impedance parameters (Z-parameter) of the transformer. It's 

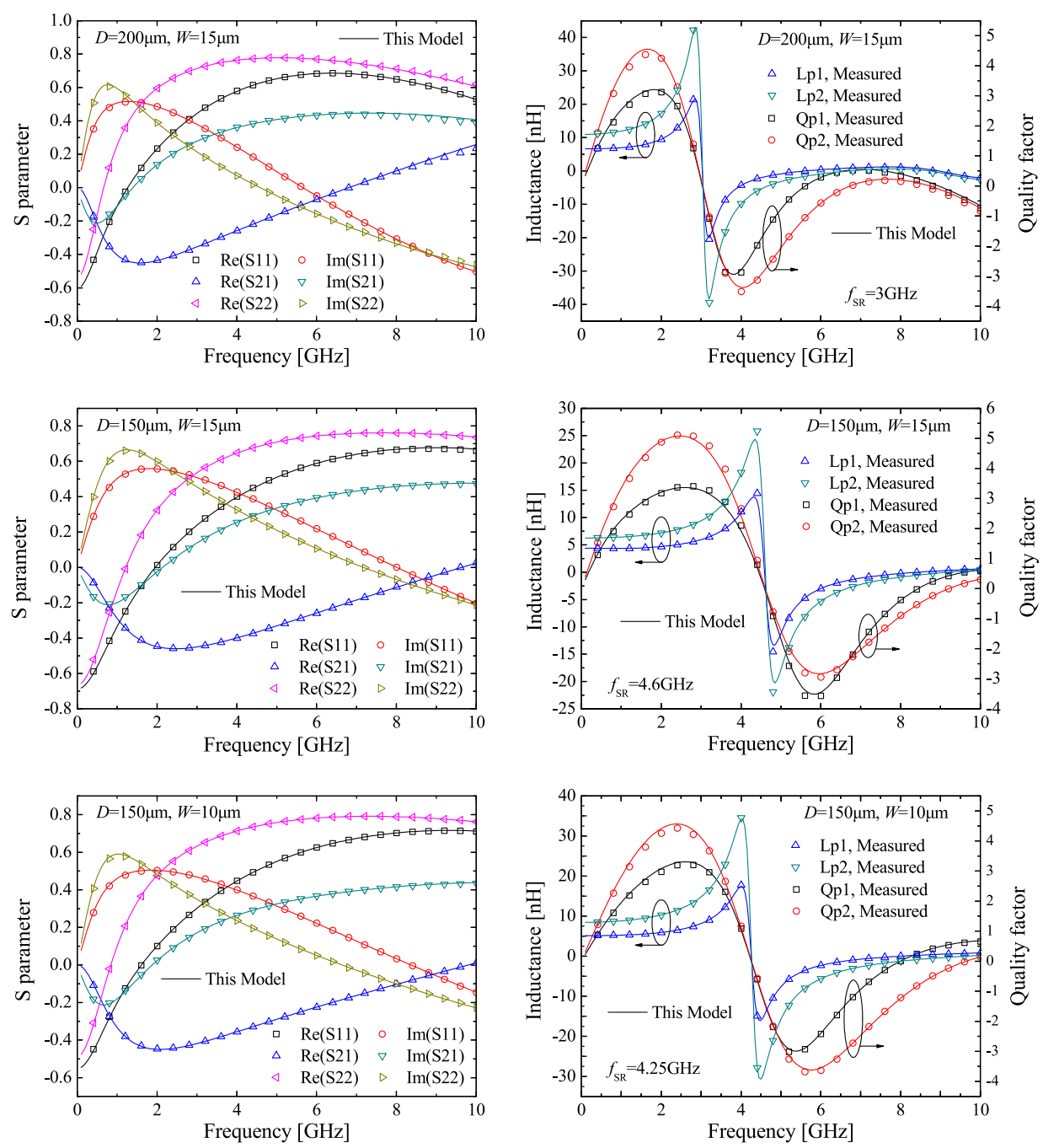

Fig. 3. Measured and fitted scattering parameters, port inductance and quality factor of (a) T1, (b) T2 and (c) T3. The subscript of "p1" and "p2" represent Port1 and Port2, respectively.

shown that the proposed model fits measured data very well. Moreover, it can be found that the model is effective for over two times self-resonance frequency.

\section{Conclusion}

A compact equivalent lumped circuit model for on-chip helical transformers is proposed. A simple coupled $R L$ loop for the skin and proximity effect and a series $R L C$ branch for the parasitic coupling effects between inductors are added into the fundamental model. Based on the measurement results, it has been verified that the proposed model is accurate for over two times self-resonance frequency, which makes it suitable for the design of high-speed and radio-frequency circuits.

\section{Acknowledgments}

This work was supported in part by the National Natural Science Foundation of China (NSFC) under Grant 61306136. 